\title{
NON-ISOMORPHIC RINGS WITH ISOMORPHIC MATRIX RINGS
}

\author{
by A. W. CHATTERS \\ (Received 17th October 1991)
}

\begin{abstract}
We construct an uncountable family of pairwise non-isomorphic rings $S_{i}$ such that the corresponding full 2 by 2 matrix rings $M_{2}\left(S_{i}\right)$ are all isomorphic to each other. The rings $S_{i}$ are Noetherian integral domains which are finitely-generated as modules over their centres.
\end{abstract}

1991 Mathematics subject classification: 16S50, 16P40, 16W20.

\section{Introduction}

Let $A$ and $B$ be rings and suppose that the corresponding full 2 by 2 matrix rings $M_{2}(A)$ and $M_{2}(B)$ are isomorphic. It is natural to ask whether this implies that $A$ and $B$ are isomorphic. The answer is well-known to be "Yes" if $A$ and $B$ are commutative, because then $A$ and $B$ are isomorphic to the centres of $M_{2}(A)$ and $M_{2}(B)$ respectively. The situation is much more interesting when $A$ and $B$ are not commutative. It was shown by S. P. Smith in [6] that there are non-isomorphic simple Noetherian integral domains $A$ and $B$ (one of which is the first Weyl algebra) such that $M_{2}(A) \cong M_{2}(B)$. Thus even for naturally-occurring Noetherian non-commutative rings it is possible to have $M_{2}(A) \cong M_{2}(B)$ without having $A \cong B$. When this happens it is probably not going to be easy to prove that $A$ and $B$ are not isomorphic, because the condition that $M_{2}(A) \cong M_{2}(B)$ implies that $A$ and $B$ are indistinguishable as far as most ring-theoretic properties and invariants are concerned. For further developments concerning Smith's example see for instance [7] and [3].

Some examples much more closely related to commutative rings than Smith's were constructed in [2]. There it was shown that, for an odd prime number $p$, the different representations of $p$ as a sum of four squares give rise to pairwise non-isomorphic rings $S$ such that the corresponding rings $M_{2}(S)$ are all isomorphic to each other. In this case the rings $S$ are Noetherian Z-orders in the division algebra of quaternions over the rational numbers. The present paper arose from an attempt to generalise the results in [2], and in particular to replace number-theoretic conditions by ring-theoretic ones. Thus the key property of the quaternion examples considered in [2] is that all relevant one-sided ideals are principal, and the condition about sums of squares can be re-stated in terms of the orbits of certain right ideals under the action of the automorphism group of the ring.

In Section 3 we shall give a general method which starts with a suitable prime ring $R$ 
and from it constructs pairwise non-isomorphic subrings $S$ such that the corresponding matrix rings $M_{2}(S)$ are all isomorphic. Roughly speaking, the subrings $S$ correspond to the orbits of the automorphism group of $R$ acting on a certain set of right ideals (the precise details are given in Theorem 3.10). As an illustration, in Section 4 we shall construct an uncountable family of non-isomorphic subrings $S$ of a ring $R$ such that the corresponding rings $M_{2}(S)$ are all isomorphic. All the rings in this example are Noetherian orders in a division algebra which is 4-dimensional over its centre. It is now becoming clear that non-isomorphic rings with isomorphic matrix rings occur quite widely. For further results about matrix rings the interested reader is strongly recommended to consult [4].

\section{Conventions and notation}

All rings considered here are associative with identity element. We shall use $\mathbf{Z}$ to denote the ring of integers. Let $R$ be a ring. For a positive integer $n$ we use $M_{n}(R)$ to denote the ring of all $n$ by $n$ matrices with entries in $R$. Let $K$ be a right ideal of $R$. We use $I_{R}(K)$ or $I(K)$ to denote the idealiser of $K$ in $R$, i.e. $I_{R}(K)=\{x \in R: x K \subseteq K\}$. Let $x \in R$. We use $r_{R}(x)$ or $r(x)$ to denote the right annihilator of $x$ in $R$, i.e. $r_{R}(x)=\{a \in R$ : $x a=0\}$. Similarly $l_{R}(x)=l(x)=\{a \in R: a x=0\}$. We say that $x$ is right regular, left regular, or regular according as $r(x)=0, l(x)=0$, or $r(x)=l(x)=0$ respectively. If $X$ is an $R$ module of finite length, we use length $(X)$ to denote the length of $X$. Background material about non-commutative rings can be found in [1] or [5].

\section{Idealisers of principal right ideals}

In this section we introduce the type of ring for which our methods work, before calculating with a particular example in Section 4. We shall also establish ways of proving that certain related rings are, or are not, isomorphic. Some standard notation will be introduced and used subsequently without further explanation.

Lemma 3.1. Let $S$ be a simple Artinian ring and let $X_{1}$ and $X_{2}$ be right ideals of $S$ such that length $\left(X_{1}\right)=$ length $\left(X_{2}\right)$. Then there is an element $u$ of $S$ with $u^{2}=1$ such that $X_{2}=u X_{1}$.

Proof. Set $Y_{3}=X_{1} \cap X_{2}$. We have $X_{1}=Y_{1} \oplus Y_{3}$ and $X_{2}=Y_{2} \oplus Y_{3}$ for some right ideals $Y_{1}$ and $Y_{2}$ of $S$ with length $\left(Y_{1}\right)=$ length $\left(Y_{2}\right)$. The sum $Y_{1}+Y_{2}+Y_{3}$ is direct, and there is a right ideal $Y_{4}$ of $S$ such that $S=Y_{1} \oplus Y_{2} \oplus Y_{3} \oplus Y_{4}$. Because length $\left(Y_{1}\right)=$ length $\left(Y_{2}\right)$, there are mutually-inverse right $S$-module isomorphisms $f: Y_{1} \rightarrow Y_{2}$ and $g$ : $Y_{2} \rightarrow Y_{1}$. Define $h: S \rightarrow S$ by $h\left(y_{1}+y_{2}+y_{3}+y_{4}\right)=g\left(y_{2}\right)+f\left(y_{1}\right)+y_{3}+y_{4}$ for all $y_{i} \in Y_{i}$. Then $h$ is a right $S$-module homomorphism, and $h^{2}$ is the identity function. Set $u=h(1)$. Then $u^{2}=h(1) h(1)=h(h(1))=1$, and $u X_{1}=h(1) X_{1}=h\left(X_{1}\right)=h\left(Y_{1}+Y_{3}\right)=f\left(Y_{1}\right)+Y_{3}=Y_{2}+Y_{3}=$ $X_{2}$.

Theorem 3.2. Let $R$ be a ring with an ideal $P$ such that the ring $R / P$ is simple 
Artinian, and let $K$ and $L$ be right ideals of $R$ which contain $P$ and satisfy length $(K /$ $P)=$ length $(L / P)$. Then $M_{2}(L)=v M_{2}(K)$ for some unit $v$ of $M_{2}(R)$, and $M_{2}(I(K)) \cong$ $M_{2}(I(L))$ as rings.

Proof. Set $S=R / P, X=K / P$, and $Y=L / P$. By Lemma 3.1 we have $Y=u X$ for some $u \in S$ with $u^{2}=1$. As in the proof of Theorem 3.3 of [2], there is a unit $v$ of $M_{2}(R)$ whose image under the natural ring-homomorphism $M_{2}(R) \rightarrow M_{2}(S)$ is

$$
\left(\begin{array}{ll}
u & 0 \\
0 & u
\end{array}\right)
$$

Because $M_{2}(Y)=u M_{2}(X)$ it follows as in the above-cited proof that $M_{2}(L)$ and $v M_{2}(K)$ have the same image in $M_{2}(\mathrm{~S})$ and hence that $M_{2}(L)=v M_{2}(K)$. We define an automorphism $f$ of $M_{2}(R)$ by $f(x)=v x v^{-1}$ for all $x \in M_{2}(R)$. Then $M_{2}(L)=f\left(M_{2}(K)\right)$. It follows that $f$ restricts to an isomorphism between the corresponding idealisers $I\left(M_{2}(L)\right)$ and $I\left(M_{2}(K)\right)$. But $I\left(M_{2}(L)\right)=M_{2}(I(L))$ and similarly for $K$. Therefore $M_{2}(I(L)) \cong M_{2}(I(K))$.

The general result in Theorem 3.2 shows that the full 2 by 2 matrix rings which we shall consider are isomorphic, but we need to work in a much more specialised situation in order to prove that the corresponding base-rings are not isomorphic.

Notation 3.3. Let $R$ be a prime ring with a non-zero central element $z$ such that $R / z R \cong M_{n}(D)$ for some division ring $D$ and positive integer $n>1$. We suppose that every one-sided ideal of $R$ which contains $z$ is principal, that $R$ has no proper non-zero idempotent two-sided ideals, and that every right or left regular element of $R$ is regular. We shall identify $R / z R$ with $M_{n}(D)$ and use ${ }^{*}$ to denote image in $R^{*}=R / z R$. We fix a right ideal $K$ of $R$ with $z R \varsubsetneqq K \varsubsetneqq R$ and set $k=\operatorname{length}\left(K^{*}\right)$. Let $L$ be the left ideal of $R$ such that $z \in L$ and $L^{*}=l_{R^{*}}\left(K^{*}\right)$, i.e. $L$ is the inverse image in $R$ of the left annihilator of $K^{*}$ in $R^{*}$. We have $K=s R$ and $L=R t$ for some $s, t \in R$.

Remarks 3.4. (1) We shall refer repeatedly to the notation and assumptions of 3.3.

(2) Because $z \in s R$ and $z$ is a non-zero central element of the prime ring $R$, in the notation of 3.3 , it is easy to show that $l(s)=0$. Thus $s$ is left regular and hence regular. Similarly $t$ is regular.

(3) It is not necessary for every one-sided ideal of $R$ to be principal in order for $R$ to satisfy 3.3. For example let $R$ be the ring of quaternions with coefficients in the ring $Z$ of integers, and let $z=p$ be any odd prime number. Then $R$ and $z$ satisfy 3.3 with $n=2$ and $D=\mathbf{Z} / p \mathbf{Z}$. But there are non-principal one-sided ideals of $R$ containing the "bad" prime 2. This example was studied in detail in [2]. 
(4) Any principal right and left ideal domain satisfies 3.3 provided that there is a suitable central element $z$, and such an example will be given in Section 4 .

(5) Once $K$ is fixed in 3.3 then $L$ is determined uniquely, but the same is not true of $s$ and $t$. For instance $t$ is only determined up to multiplication on the left by a unit of $R$. Hence $t R$ is only determined up to conjugation by a unit of $R$, but this is enough for our purposes because we are only interested in the isomorphism-type of the idealiser $I(t R)$.

(6) In the quaternion example in (3) above there is a natural choice for $t$ given $s$, namely $t$ is the usual conjugate of $s$.

(7) For varying choices of $K$ in 3.3 we wish to determine when the corresponding idealisers $I(K)$ are isomorphic. It follows from Theorem 3.2 that the rings $M_{2}(I(K))$ are isomorphic to each other for all choices of $K$ corresponding to a fixed value of $k=$ length $\left(K^{*}\right)$.

Lemma 3.5. In the context of 3.3 suppose that $z=a b$ for some $a, b \in R$. Then $z=b a$.

Proof. We have $l(a)=0$ because $l(z)=0$. Thus $a$ is left regular and hence regular. But $a b a=z a=a z$. Therefore $b a=z$.

Lemma 3.6. Let $R$ be as in 3.3. Then $z \in t R$, length $\left(t^{*} R^{*}\right)=\operatorname{leng} \operatorname{th}\left(R^{*} t^{*}\right)=n-k$, and $t s R=z R$.

Proof. Because $z \in L$ we have $z=r t$ for some $r \in R$. Hence $z=t r$ by Lemma 3.5 so that $z \in t R$. Because $R^{*}$ is simple Artinian and length $\left(K^{*}\right)=k$, it is well-known that length $\left(R^{*} t^{*}\right)=$ length $\left(l_{R^{*}}\left(K^{*}\right)\right)=n-k$ and that length $\left(t^{*} R^{*}\right)=\operatorname{length}\left(R^{*} t^{*}\right)$. Hence length $(R / t R)=$ length $\left(R^{*}\right)$ - length $\left(t^{*} R^{*}\right)=n-(n-k)=k$. Also because $t$ is regular we have $t R / t s R \cong R / s R$ as right $R$-modules. Thus length $(t R / t s R)=\operatorname{length}(R / s R)=$ length $\left(R^{*}\right)-$ length $\left(s^{*} R^{*}\right)=n-k$. But $\quad t s R \subseteq z R$ and length $(R / t s R)=\operatorname{length}(R / t R)+$ length $(t R / t s R)=k+(n-k)=n=$ length $(R / z R)$. Therefore $t s R=z R$.

Remark 3.7. Let $R$ be as in 3.3. By Lemma 3.6 we have $z=t s w$ for some unit $w$ of $R$. We have $s w R=s R=K$. Thus if we replace $s$ by $s w$ we may suppose without loss of generality that $z=t s$. By Lemma 3.5 we also have $z=s t$. From now on we shall suppose that $z=t s=s t$.

Proposition 3.8. With the assumptions of 3.3 and 3.7, there is a ring-isomorphism $f$ : $I(s R) \rightarrow I(t R)$ such that $f(R t)=t R$.

Proof. Let $x \in I(s R)$. Then $x s=s y$ for some $y \in R$. In fact $y$ is unique because $s$ is regular. We shall show that $y \in I(t R)$. We have $s y t=x s t=x z=z x=s t x$. Hence $y t=t x$ and $y \in I(t R)$. With $x s=s y$ as above, we define $f: I(s R) \rightarrow I(t R)$ by $f(x)=y$, i.e. $f(x)=s^{-1} x s$. Similarly we can define $g: I(t R) \rightarrow I(s R)$ by $g(y)=t^{-1} y t$ for all $y \in I(t R)$. 
It is routine to check that $f$ and $g$ are mutually-inverse ring-isomorphisms. Also $f(R t)=s^{-1} R t s=s^{-1} R z=z s^{-1} R=t s s^{-1} R=t R$.

Proposition 3.9. With the assumptions of 3.3 and 3.7, then $s R$ and $R t$ are the only idempotent maximal ideals of $I(s R)$. Also $I(s R) / s R \cong M_{n-k}(D)$ and $I(s R) / R t \cong M_{k}(D)$.

Proof. It is well-known and easy to show that $I(s R) / s R$ is isomorphic to the endomorphism ring $\operatorname{End}_{R}(R / s R)$ of the right $R$-module $R / s R$. But $R / s R \cong R^{*} / s^{*} R^{*}$. Also $R^{*}=M_{n}(D) \quad$ and length $\left(R^{*} / s^{*} R^{*}\right)=n-k$. Therefore $I(s R) / s R \cong \operatorname{End}_{R}(R / s R) \cong$ $\operatorname{End}_{R^{*}}\left(R^{*} / s^{*} R^{*}\right) \cong M_{n-k}(D)$. Similarly $I(t R) / t R \cong M_{k}(D)$, because length $\left(R^{*} / t^{*} R^{*}\right)=$ length $\left(R^{*}\right)-$ length $\left(t^{*} R^{*}\right)=n-(n-k)=k$ by Lemma 3.6. But by Proposition 3.8 we know that $I(t R) / t R \cong I(s R) / R t$. Therefore $I(s R) / R t \cong M_{k}(D)$. In particular it follows that $R t$ and $s R$ are maximal ideals of $I(s R)$. Because $z R \varsubsetneqq s R$ and $z R$ is a maximal ideal of $R$, we have $R s R=R$. Therefore $s R$ is idempotent, and similarly so also is $R t$.

Now let $M$ be a maximal ideal of $I(s R)$ with $s R \neq M \neq R t$. We must show that $M$ is not idempotent. Suppose that $R M R=R$; we shall obtain a contradiction. We have $s R \cdot R t=s R t=s R M R t \subseteq M$. Hence either $s R \subseteq M$ in which case $s R=M$, or $R t \subseteq M$ giving $R t=M$. This is a contradiction, so that we have $R M R \neq R$. Therefore $R M R$ is not idempotent, by 3.3. Thus $R M R \supsetneqq(R M R)^{2} \supseteqq R M^{2} R$. It follows that $M^{2} \neq M$.

We have shown in Proposition 3.9 that $s R$ and $R t$ can be distinguished from all other ideals of $I(s R)$ because they are the only idempotent maximal ideals. The proof of the following result depends heavily on this fact. We have $R t \neq s R$ for otherwise $s R=R s R=R$.

Theorem 3.10. Let $R, z, s, t$ be as in 3.3 and 3.7. Also let $u$ be an element of $R$ such that $z R \varsubsetneqq u R \varsubsetneqq R$ and length $\left(u^{*} R^{*}\right)=k=$ length $\left(s^{*} R^{*}\right)$, and let $v$ correspond to $u$ in the same way that $t$ corresponds to $s$. Suppose that there is a ring-isomorphism $f$ : $I(s R) \rightarrow I(u R)$. Then there is a ring-automorphism $g$ of $R$ with $g(z R)=z R$ such that one of the following is true:

(1) $f$ is the restriction of $g$ to $I(s R)$ and $g(s R)=u R$, or

(2) $g(s R)=v R$ and $2 k=n$.

Proof. Set $S=I(s R)$ and $U=I(u R)$. By Proposition 3.9 we know that $s R$ is an idempotent maximal ideal of $S$. Hence $f(s R)$ is an idempotent maximal ideal of $U$. But $u R$ and $R v$ are the only idempotent maximal ideals of $U$, by Proposition 3.9. Therefore either $f(s R)=u R$ or $f(s R)=R v$.

Case (1): Suppose that $f(s R)=u R$. We shall show that $f$ lifts to an automorphism of $R$. Because $R t$ and $R v$ are the only other idempotent maximal ideals of $S$ and $U$ respectively (Proposition 3.9), we have $f(R t)=R v$. Hence $f(z R)=f(R t s R)=$ $f(R t) f(s R)=R v u R=z R$. Set $w=f(z)$. We wish to show that $w R=z R$. We have $w \in z R$ so that $w=z a$ for some $a \in R$. But $z$ is central in $R$ and $S$ and hence $w$ is central in $U$. Thus $z a u=w u=u w=u z a=z u a$ so that $a u=u a$. Hence $a \in U$. Similarly by using the isomor- 
phism $f^{-1}: U \rightarrow S$ we see that $f^{-1}(z)=z b$ for some $b \in S$. Set $c=f(b)$. Then $c \in U$ and $z=f(z b)=f(z) f(b)=w c$. Hence $z R=w c R \subseteq w R$. Because $w=f(z) \in z R$ it follows that $z R=w R$ as required.

Now for $x \in R$ we have $f(z x) \in f(z R)$ with $f(z R)=z R=w R$. Thus $f(z x)=w y$ for some $y \in R$. Set $g(x)=y$, i.e. $g(x)=w^{-1} f(z x)$ for all $x \in R$. It is straightforward to check that $g$ is an automorphism of $R$. For example if $p, q \in R$ then $g(p q)=w^{-1} f(z p q)=$ $w^{-2} f(z) f(z p q)=w^{-2} f\left(z^{2} p q\right)=w^{-2} f(z p) f(z q)=w^{-1} f(z p) w^{-1} f(z q)=g(p) g(q) ; \quad$ here we have used the fact that $w$ is central in $U$. Also for $x \in S$ we have $g(x)=w^{-1} f(z x)=$ $w^{-1} f(z) f(x)=f(x)$.

Case (2): Suppose that $f(s R)=R v$. Then $f: S \rightarrow U$ induces an isomorphism between $S / s R$ and $U / R v$. But by Proposition 3.9 we have $S / s R \cong M_{n-k}(D)$ and $U / R v \cong M_{k}(D)$. Therefore $M_{n-k}(D) \cong M_{k}(D)$, i.e. $n-k=k$, i.e. $2 k=n$. Set $V=I(v R)$. By Proposition 3.8 there is an isomorphism $h: U \rightarrow V$ such that $h(R v)=v R$. Thus $h f: S \rightarrow V$ is an isomorphism with $h f(s R)=h(R v)=v R$. Now we can use what was proved in (1) to deduce that $h f$ extends to an automorphism $g$ of $R$ with $g(z R)=z R$ and $g(s R)=v R$.

Corollary 3.11. In the notation of 3.10 suppose that $k \neq n / 2$ and that $f: I(s R) \rightarrow I(u R)$ is a ring-isomorphism. Then $f$ extends to an automorphism of $R$.

Proof. This is an immediate consequence of Theorem 3.10, because possibility (2) is ruled out by the assumption that $k \neq n / 2$.

It would be nice if (2) of Theorem 3.10 never occurred, and certainly it does not occur if $n$ is odd. However, even for $n=3$, the calculations in a particular example can be very complicated. The easiest examples to work with have $n=2$, but then Theorem 3.10(1) may fail as in the following example.

Example 3.12. As in Example 3.8 of [2], let $R$ be the ring of quaternions with integer coefficients, and take $z=71, s=1+3 i+5 j+6 k$, and $u=1-3 i-5 j-6 k$. Then $u s=s u=z$, so that we can take $t=u$. By Proposition 3.8 we have $I(s R) \cong I(u R)$. We are in the situation of Theorem 3.10 with $n=2, k=1$, and $D=Z / 71 Z$. But it was shown in 3.8 of [2] that there is no automorphism $g$ of $R$ such that $g(s R)=u R$. Thus Theorem $3.10(1)$ fails.

\section{The example}

We shall now look in detail at the example which will provide an uncountable family of pairwise non-isomorphic rings $S$ such that the corresponding 2 by 2 matrix rings $M_{2}(S)$ are all isomorphic to each other.

Notation 4.1. $C$ denotes the field of complex numbers; for $c \in C$ we use $\bar{c}$ and real $(c)$ to denote respectively the complex conjugate of $c$ and the real part of $c ; R$ is the ring of polynomials in $X$ over $C$ with multiplication twisted by the rule that $X c=\bar{c} X$ for all $c \in C ; z=X^{4}+1$. 
The notation of 4.1 will be used through the rest of this section. It is well-known that $R$ is an integral domain in which every one-sided ideal is principal (see for example [5] 1.2.9). Clearly $z$ is a central element of $R$. It is known that $R / z R \cong M_{2}(C)$, and we shall prove this in 4.5 as a consequence of studying those right ideals of $R$ which contain $z$. Thus we are in the situation of Section 3 with $n=2$. We shall also need to look at the automorphisms of $R$, and the word "automorphism" here will always mean "ring-automorphism".

Proposition 4.2. With the notation of 4.1 let $g$ be an automorphism of $R$. Then $g(X)=a X$ for some non-zero element $a$ of $C$. Also the restriction of $g$ to $C$ is either the identity function or complex conjugation.

Proof. The non-zero elements of $C$ are the units of $R$. From this it follows that $g$ restricts to an automorphism of $C$. Also $g(X)$ generates $R$ as a $C$-algebra, so that $g(X)=a X+b$ for some $a, b \in C$ with $a \neq 0$. For all $c \in C$ we have $g(X c)=g(\bar{c} X)$, so that $(a X+b) g(c)=g(\vec{c})(a X+b)$. In particular $g(c) b=g(\bar{c}) b$ for all $c \in C$. Hence $b=0$. Thus for all $c \in C$ we have $a X g(c)=g(\bar{c}) a X$, i.e. $(\overline{g(c)}) a=g(\bar{c}) a$, i.e. $(\overline{g(c)})=g(\bar{c})$. Therefore $g(c)$ is a real number if and only if $c$ is real. Hence the restriction of $g$ to the real numbers is an automorphism and consequently is the identity function. It follows from this that the restriction of $g$ to $C$ is either the identity function or complex conjugation.

Corollary 4.3. Let $g$ be an automorphism of $R$ such that $g(z R)=z R$. Then $g(X)=a X$ for some $a \in C$ with $|a|=1$.

Proof. By Proposition 4.2 we have $g(X)=a X$ for some $a \in C$ with $a \neq 0$. Thus $g(z)=g\left(X^{4}+1\right)=(g(X))^{4}+1=(a X)^{4}+1=(a \bar{a})^{2} X^{4}+1=|a|^{4} X^{4}+1$. But $z R=g(z R)=g(z) R$. Hence $z=g(z) u$ for some unit $u$ of $R$, i.e. for some non-zero element $u$ of $C$. Thus $X^{4}+1=\left(|a|^{4} X^{4}+1\right) u$. Therefore $u=1$ and $|a|=1$.

Proposition 4.4. Let $K$ be a right ideal of $R$ with $z R \varsubsetneqq K \varsubsetneqq R$. Then $K=$ $\left(X^{2}+a X+b\right) R$ for some $a, b \in C$ with $|b|=1$ and $|a|^{2} / 2=\operatorname{real}(b)$. Conversely if $a$ and $b$ are any such elements of $C$ and $K=\left(X^{2}+a X+b\right) R$, then $z R \varsubsetneqq K \varsubsetneqq R$.

Proof. Firstly suppose that $K$ is a right ideal of $R$ with $z R \varsubsetneqq K \varsubsetneqq R$. Because every one-sided ideal of $R$ is principal we have $K=s R$ for some $s \in R$. Thus $z=s t$ for some $t \in R$. If $r \in R$ let $\operatorname{deg}(r)$ denote the degree of $r$ as a polynomial in $X$. Then $\operatorname{deg}(s)+\operatorname{deg}(t)=\operatorname{deg}(z)=4$. Because $z R \varsubsetneqq s R \varsubsetneqq R$ we have $4 \neq \operatorname{deg}(s) \neq 0$. Therefore $1 \leqq$ $\operatorname{deg}(s) \leqq 3$. Without loss of generality we can suppose that $s$ is monic.

In order to obtain a contradiction we suppose that $\operatorname{deg}(s)=1$, i.e. that $s=X+c$ for some $c \in C$. Then $t=X^{3}+u X^{2}+v X+w$ for some $u, v, w \in C$. Because $X^{4}+1=z=s t=$ $(X+c)\left(X^{3}+u X^{2}+v X+w\right)$ we have $0=c+\bar{u}=c u+\bar{v}=c v+\bar{w}$ and $1=c w$. Hence $1=c w=$ $c(\overline{-c v})=-c \bar{c} \bar{v}=c^{2} \bar{c} u=-(c \bar{c})^{2}$, i.e. $1=-|c|^{4}$. This is a contradiction. Hence $\operatorname{deg}(s) \neq 1$. Similarly $\operatorname{deg}(t) \neq 1$, i.e. $\operatorname{deg}(s) \neq 3$.

Therefore $\operatorname{deg}(s)=2$, i.e. $s=X^{2}+a X+b$ for some $a, b \in C$. Also $t=X^{2}+p X+q$ for 
some $p, q \in C$. Because $X^{4}+1=\left(X^{2}+a X+b\right)\left(X^{2}+p X+q\right)$ we have $0=p+a=q+a \bar{p}+$ $b=a \bar{q}+b p$ and $1=b q$. Hence $p=-a$ and $0=a \bar{q}+b p=a(\bar{q}-b)$. Thus either $a=0$ or $b=\bar{q}$. If $a=0$ then $0=q+a \bar{p}+b=q+b$, so that $1=b q=-b^{2}$ and $b= \pm i$. Now suppose that $a \neq 0$. Then $b=\bar{q}$ so that $1=b q=b \bar{b}$. Hence $|b|=1$ and $0=q+a \bar{p}+b=\bar{a}-a \bar{a}+b=2$ $\operatorname{real}(b)-|a|^{2}$.

Conversely let $a, b \in C$ with $|b|=1$ and $|a|^{2} / 2=\operatorname{real}(b)$. Set $s=X^{2}+a X+b$ and $t=X^{2}-a X+b$. Then $s t=z$ from which it follows easily that $z R \varsubsetneqq s R \varsubsetneqq R$.

Corollary 4.5. $R / z R \cong M_{2}(C)$.

Proof. Let $e_{p q}$ denote the 2 by 2 matrix with 1 in the $(p, q)$-position and 0 's elsewhere. Set $f(X)=i e_{12}+e_{21}$ where $i^{2}=-1$ in $C$, and for each $c \in C$ set $f(c)=$ $c e_{11}+\bar{c} e_{22}$. Then $f(X) f(c)=f(\bar{c}) f(X)$ for all $c \in C$. Therefore $f$ can be extended to a ring homomorphism from $R$ to $M_{2}(C)$. It follows from 4.4 that $\operatorname{Ker}(f)=z R$. Also $\operatorname{deg}(z)=4$ so that $R / z R$ has dimension 4 as a vector space over $C$. It follows that $f(R)=M_{2}(C)$ and that $R / z R \cong M_{2}(C)$

Corollary 4.6. Let $K$ be a right ideal of $R$ with $z R \varsubsetneqq K \varsubsetneqq R$. In the notation of 3.3 we have $n=2$ and $k=1$. Also we can take $K=s R$ and $L=R$ there $s=X^{2}+a X+b$ and $t=X^{2}-a X+\bar{b}$ for some $a, b \in C$ with $|b|=1$ and $|a|^{2} / 2=\operatorname{real}(b)$.

The next result will enable us to avoid the awkward Case (2) of Theorem 3.10.

Lemma 4.7. Let $a, b \in C$ with $|b|=1$ and $|a|^{2} / 2=\operatorname{real}(b)$. Set $s=X^{2}+a X+b$ and $t=X^{2}-a X+b$. Then there is an automorphism $g$ of $R$ such that $g(z R)=z R$ and $g(s R)=t R$.

Proof. If $a=0$ set $g(X)=X$; if $a \neq 0$ set $g(X)=(-a / \bar{a}) X$; set $g(c)=\bar{c}$ for all $c \in C$. Then $g$ can be extended to an automorphism of $R$ with the desired properties. In fact $g(s)=t$, $g(t)=s$, and $g(z)=z$.

Theorem 4.8. Let $R$ be as in 4.1, and let $a, b, c, d \in C$ with $|b|=1=|d|,|a|^{2} / 2=\operatorname{real}(b)$, $|c|^{2} / 2=\operatorname{real}(d)$. Set $s=X^{2}+a X+b$ and $u=X^{2}+c X+d$. Then

(a) $M_{2}\left(I_{R}(s R)\right) \cong M_{2}\left(I_{R}(u R)\right)$, and

(b) The following statements are equivalent:

(1) $I_{R}(s R) \cong I_{R}(u R)$ as rings;

(2) $\operatorname{real}(b)=\operatorname{real}(d)$;

(3) $u R=g(s R)$ for some automorphism $g$ of $R$.

Proof. (a) follows from Proposition 4.4, Corollary 4.5 and Theorem 3.2.

(b) $(3) \Rightarrow(1)$ is elementary.

$(1) \Rightarrow(2)$ : Suppose that $I(s R) \cong I(u R)$ and set $v=X^{2}-c X+d$. By Corollary 4.6 and 
Theorem 3.10 there is an automorphism $g$ of $R$ with $g(z R)=z R$ such that either $g(s R)=u R$ or $g(s R)=v R$. Also by Lemma 4.7 there is an automorphism $h$ of $R$ such that $h(z R)=z R$ and $h(u R)=v R$. Therefore there is an automorphism $f$ of $R$ such that $f(z R)=z R$ and $f(s R)=u R$. By Proposition 4.2 and Corollary 4.3 we have $f(X)=w X$ for some $w \in C$ with $|w|=1$, and the restriction of $f$ to $C$ is either the identity function or complex conjugation. We have $f(s)=f\left(X^{2}+a X+b\right)=(w X)^{2}+f(a) w X+f(b)=X^{2}+$ $f(a) w X+f(b)$. Also $f(s R)=u R$, i.e. $\left(X^{2}+f(a) w X+f(b)\right) R=\left(X^{2}+c X+d\right) R$. Hence $X^{2}+f(a) w X+f(b)=X^{2}+c X+d$. In particular this gives $d=f(b)$ where $f(b)=b$ or $f(b)=\bar{b}$. Therefore real $(d)=\operatorname{real}(b)$.

(2) $\Rightarrow(3)$ : Suppose that $\operatorname{real}(b)=\operatorname{real}(d)$. Because $|b|=1=|d|$ and real $(b)=\operatorname{real}(d)$ we have $d=b$ or $d=b$. We must show that $u R=g(s R)$ for some automorphism $g$ of $R$, and we shall do this by considering various cases. Suppose firstly that $a=0$. Then $|c|^{2} / 2=$ real $(d)=$ real $(b)=|a|^{2} / 2=0$ so that $c=0$. If $d=b$ we can take $g$ to be the identity function on $R$. If $d=\bar{b}$ we can take $g$ to be the automorphism of $R$ determined by $g(X)=X$ and $g(p)=\bar{p}$ for all $p \in C$.

Now assume that $a \neq 0$. Then we also have $c \neq 0$. If $d=b$ we can take $g$ to be the automorphism of $R$ determined by $g(X)=(c / a) X$ and $g(p)=p$ for all $p \in C$. If $d=\bar{b}$ we take $g(X)=(c / \bar{a}) X$ and $g(p)=\bar{p}$ for all $p \in C$. In all cases we have determined an automorphism $g$ of $R$ such that $g(s R)=u R$.

Corollary 4.9. Let $A$ and $B$ be maximal right ideals of $R$ which contain $z$. Then $M_{2}(I(A)) \cong M_{2}(I(B))$, but $I(A) \cong I(B)$ if and only if $B=g(A)$ for some automorphism $g$ of $R$.

Proof. This follows from Theorem 3.2, Proposition 4.4 and Theorem 4.8.

Corollary 4.10. Set $I=\{x: x$ is a real number with $0 \leqq x \leqq 1\}$. For each $p \in I$ set $\left.K_{p}=\left(X^{2}+(2 p)^{1 / 2} X+p+i(1-p)^{2}\right)^{1 / 2}\right) R$. Then $K_{p}$ is a maximal right ideal of $R$ containing z. As $p$ ranges over the elements of $I$ the corresponding uncountable family of rings $I_{R}\left(K_{p}\right)$ are pairwise non-isomorphic, but the matrix rings $M_{2}\left(I_{R}\left(K_{p}\right)\right)$ are all isomorphic to each other.

Proof. By Proposition 4.4 each $K_{p}$ is a maximal right ideal of $R$ containing $z$. Let $p$, $q \in I$. It follows from Theorem 4.8 that the three following statements are equivalent: $I\left(K_{p}\right) \cong I\left(K_{q}\right) ; \operatorname{real}\left(p+i\left(1-p^{2}\right)^{1 / 2}\right)=\operatorname{real}\left(q+i\left(1-q^{2}\right)^{1 / 2}\right) ; p=q$. We have $M_{2}\left(I\left(K_{p}\right)\right) \cong$ $M_{2}\left(I\left(K_{q}\right)\right)$ by Theorem 3.2 .

\section{REFERENCES}

1. A. W. Chatters and C. R. Hajarnavis, Rings with chain conditions (Pitman, London, 1980).

2. A. W. Chatters, Matrices, idealisers, and integer quaternions, J. Algebra, 150 (1992), 45-56.

3. G. Letzter and L. Makar-Limanov, Rings of differential operators over rational affine curves, Bull. Soc. Math. France, to appear.

4. L. S. Levy, J. C. Robson and J. T. Stafford, Hidden matrices, in preparation. 
5. J. C. McConnell and J. C. Robson, Non-commutative Noetherian rings (Wiley, 1987).

6. S. P. Smith, An example of a ring Morita-equivalent to the Weyl algebra $A_{1}, J$. Algebra 73 (1981), 552-555.

7. J. T. Stafford, Endomorphisms of right ideals of the Weyl algebra, Trans. Amer. Math. Soc. 299 (1987), 623-639.

School of Mathematics

UNIVERSITY WALK,

BRISTOL BS8 1TW 